\title{
Correlated states and nuclear reactions: An experimental test with low energy beams
}

\author{
Sergio Bartalucci, ${ }^{1, *}$ V. I. Vysotskii, ${ }^{2}$ and M. V. Vysotskyy ${ }^{2}$ \\ ${ }^{1}$ INFN Laboratori Nazionali di Frascati, Frascati, 00044 Italy \\ ${ }^{2}$ Taras Shevchenko National University of Kyiv, Kyiv, 01601 Ukraine
}

(Received 30 November 2018; published 31 May 2019)

\begin{abstract}
An experimental program is described in this paper, aiming at detecting the formation of correlated coherent states (CCSs) in thin surface layers of crystals when bombarded by a very low energy proton or deuteron beam. CCSs are a generalization of "nonclassical" states of light, such as coherent and squeezed states, whose existence has been demonstrated long ago, giving rise to the remarkable development of quantum optics. In other fields, ranging from condensed matter physics to cosmology, such states have been intensively studied, but a clear signature of their existence is still lacking. This may be a clue to several unexplained phenomena, including the strong enhancement of nuclear fusion reaction rates in some crystal lattices, which have been reported on by several experiments and cannot be accounted for by electron screening only. Such an investigation is extremely relevant to nuclear astrophysics studies, few-body nucleon systems, and nucleon nucleon-interaction problems and, in particular, to energy-related nuclear fusion studies.
\end{abstract}

DOI: $10.1103 /$ PhysRevAccelBeams.22.054503

\section{INTRODUCTION}

A strong enhancement of the sub-barrier nuclear fusion cross section has been reported in the recent past by several experiments [1], which cannot be accounted for by the usual electron screening effect. In particular, reactions occurring at a very low energy in various metallic environments, that can simulate a dense, strongly coupled astrophysical plasma, show, besides a strong dependence on the target material, other surprising features, such as branching ratio alterations and increased angular distribution anisotropy, which were not observed in gas targets. Energy spread of either the target or impinging nuclei is deemed to be responsible for these effects, although the possible generating mechanisms, such as thermal motion, vibrations inside an atomic, molecular, or crystal system, or a finite beam energy width, seem quite negligible at energies down to $\approx 10 \mathrm{KeV}$. Other groups [2] have reported on a strong screening enhancement of the D-D fusion reactions in some metals, but the presence of narrow threshold resonances, as suggested by the authors, seems not a sufficient explanation. In particular, a team at the ECRIS facility of the University of Stettin is planning to

\footnotetext{
*Sergio.Bartalucci@lnf.infn.it

Published by the American Physical Society under the terms of the Creative Commons Attribution 4.0 International license. Further distribution of this work must maintain attribution to the author(s) and the published article's title, journal citation, and DOI.
}

make measurements at deuteron energies below $5 \mathrm{keV}$ for a better understanding of the observed phenomena [3].

A very intriguing effect has been recently observed by an independent group [4], which used a low energy proton beam and a thin crystalline lithium target (foil) or nanocrystalline $(\mathrm{Li})_{N}$ fragments formed by the evaporation of lithium. In these experiments, a big bump in the counting rate of produced fast alpha particles was found at an energy of about $8.6 \mathrm{MeV}$, thereby providing evidence of the occurring reaction ${ }^{7} \mathrm{Li}(p, \alpha){ }^{4} \mathrm{He}(Q=17.35 \mathrm{MeV})$. At the same time, neither alpha nor other nuclear particles were detected at a lower energy, showing in turn a total absence of the reaction involving the lighter isotope ${ }^{6} \mathrm{Li}(p ., \alpha)^{3} \mathrm{He}(Q=4.02 \mathrm{MeV})$.

The maximum rate occurred with protons incident on the target at an energy of about $500 \mathrm{eV}$, which is just the order of magnitude of the energy at which the formation of special quantum states, named correlated coherent states (CCSs) [5-7], is expected, as is shown in Ref. [5]. The possibility of performing cross-section measurements at such energies (below $1 \mathrm{keV}$ ) is the main reason for this experiment.

A different approach was envisaged in some theoretical studies, suggesting a possible huge increase of the tunneling of quantum packets through a high Coulomb barrier, owing either to a better evaluation of the transmission coefficient as compared to the standard (WKB) one [6] or to the formation of CCSs. In both cases, the impinging particle momentum spread is shown to play a crucial role, even if much smaller than the central momentum.

In this paper, some theoretical introduction of the latter model is given, and a principle experimental approach is 
described. The importance of such an investigation is to be found not only in a plethora of nuclear, astrophysical, and cosmological studies, but also in the development of new energy-producing systems, including low-energy nuclear reactions.

\section{THEORETICAL PREMISES}

The Heisenberg uncertainty relation (HUR) for position and momentum operators [8], as put in the usual form by Kennard [9], $\Delta p \Delta q \geq \hbar / 2$, is a particular case of a more general formulation, the Schrödinger-Robertson inequality (SRI) $[10,11]$, which takes into account also the possibility of a correlation between the two physical observables:

$$
\Delta p \Delta q \geq \sqrt{\left(\frac{1}{2}\langle\{p, q\}\rangle-\langle p\rangle\langle q\rangle\right)^{2}+\left|\frac{1}{2 i}\langle[p, q]\rangle\right|^{2}} .
$$

The first term under the square root sign is the first-order covariance (or correlation) between the two observables, as represented by the average half-value of their anticommutator minus the product of their average values, while the second term is the usual commutator which must fulfill the quantization postulate $[p, q]=p q-q p=-i \hbar$. Introducing a correlation (or covariance) coefficient $r=(1 / 2\langle\{p, q\}\rangle-\langle p\rangle\langle q\rangle) / \Delta p \Delta q$, the SRI can be written as $(\Delta p)^{2}(\Delta q)^{2} \geq \hbar^{2} /\left[4\left(1-r^{2}\right)\right]$ or, equivalently, in the more usual notation $\sigma_{p} \sigma_{x} \geq \hbar / 2 \sqrt{\left(1-r^{2}\right)}$. A similar modified formula corresponds to the ratio of energy and time (see, e.g., $[12,13])$. The question about the existence of quantum states for which the sign $\geq$ may become = exactly (i.e., the minimum uncertainty states) remained unanswered until the early 1960s, when high-power coherent microwave and light sources, such as masers and lasers, were invented and gave rise to the remarkable development of quantum optics. First, the existence of coherent states, depending on two real parameters, which can minimize the HUR was suggested [14] and experimentally proved, and then the "squeezed" states depending on a further parameter, the squeezing factor $k=\Delta p / \Delta q$, were experimentally found by several researchers [15].

They can be useful, e.g., in minimizing the quantum (shot) noise in special applications such as gravitational wave interferometry.

At the same time, a further generalization was introduced, the CCSs, which equalize the SRI so that they must be considered as the minimum uncertainty states in the Schrödinger-Robertson sense. They were analytically constructed and their eigenfunctions evaluated by Dodonov, Klimov, and Man'ko [16] ( $\beta$ is a complex number) as

$\Psi_{\beta}\left(x \mid r, \sigma_{q}\right)=N_{\beta} \exp \left[-\frac{x^{2}}{4 \sigma_{q}^{2}}\left(1-\frac{i r}{\sqrt{1-r^{2}}}\right)+\frac{\beta x}{\sigma_{q}}\right]$.

A graphical representation of these states in a phase space diagram is depicted in Fig. 1.

In the same paper, it was suggested that, in the case of full correlation $(r \Rightarrow 1)$, huge values of momentum and
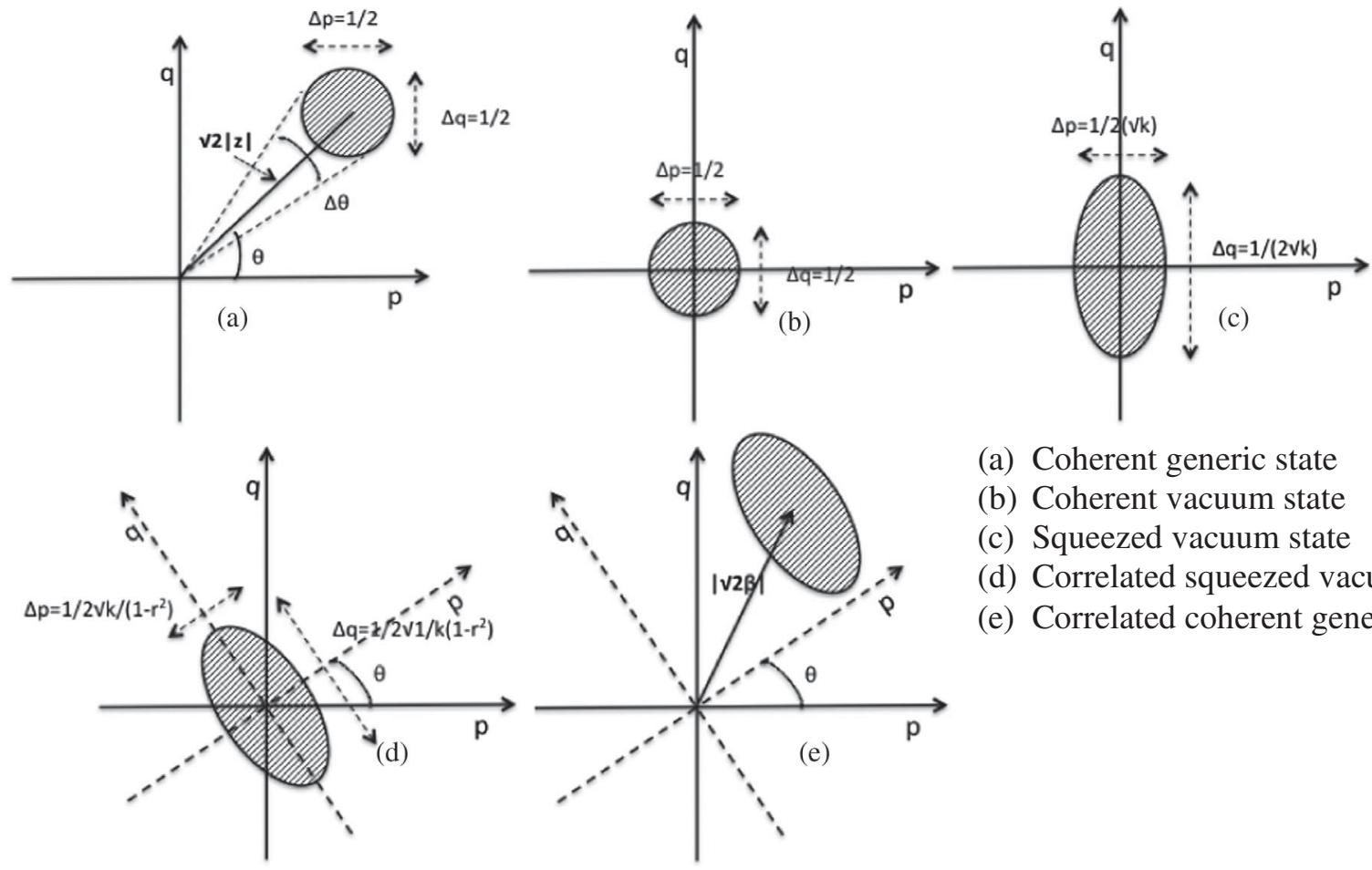

(a) Coherent generic state

(b) Coherent vacuum state

(c) Squeezed vacuum state

(d) Correlated squeezed vacuum state

(e) Correlated coherent generic state

FIG. 1. Uncertainty areas in phase space: a) coherent generic state, b) coherent vacuum state, c) squeezed vacuum state, d) correlated squeezed vacuum state, e) correlated coherent generic state. 
position dispersions can be obtained, so the formation of CCSs can lead to an enormous increase of Coulomb barrier transparency.

The physical reason for this is the following: If a particle is in a superposition of uncorrelated states, its probability of tunneling through the barrier is just the sum of probabilities for each independent state, which is, in general, very low. But when the particle is in a superposition of partially correlated states, this can lead to a partial mutual compensation of reflected waves from the potential well, i.e., to a destructive interference of reflected waves. At full correlation, full suppression of reflected waves, and hence full transmissivity, may occur. This effect is due to the synchronization of a large number of momentum fluctuations, not to the increase of the particle's average momentum and average kinetic energy.

An additional fundamental difference of the tunneling process for particles with "real" energy, obtained, for example, during "usual" acceleration, from the case of "virtual" energy, generated during the formation of a coherent correlated state is that the fluctuation of large virtual energy exists for a short time. This automatically excludes those reactions whose duration significantly exceeds this time. This issue will be discussed below.

It must be stressed, however, that the increase of the product of momentum and position dispersion is not an automatic guarantee for an improvement of barrier transparency: It depends much on specific cases. While referring to the existing literature for full details [17], it must be noted that, even using a different approach to the transmissivity problem of low energy particles, other authors arrive at quite a similar result without invoking the formation of CCSs [6]. According to this last analysis, the transmissivity of a slow quantum packet through the high Coulomb barrier may increase exponentially according to the following equation $(a=$ const):

$$
T_{*} \sim \exp \left[-(3 / 2)\left(a^{2} / \sigma_{p}\right)^{1 / 3}\right]=[T(r=0)]^{\left(1-r^{2}\right)^{1 / 3}} .
$$

In the alternative problem of particle transmission in the form of a plane wave, this probability qualitatively coincides with Eq. (2) but with the replacement of the exponent $1 / 3$ by $1 / 2[5,17]$. Such a difference appears because the wave packet is a superposition of plane waves characterizing the motion of the particle rather than a single wave. Some of these waves can have high momentum and energy; most of the other components of the packet have low momentum and energy, and this probability for them will decrease sharply. CCS formation should be observed in a nonstationary harmonic oscillator, in the parabolic field of which the particle under investigation is located.

Various mechanisms of parametric or externally driven frequency modulation, such as a monotonic asymptotic decrease or increase in the oscillator frequency, or the variation of this frequency in a bounded interval or periodic modulation in the absence or presence of a random force as well as in the case of a fluctuation of the modulation frequency can lead to the optimal mutual phasing of different eigenstates of the particle in this well.

In fact, the explicit form of the CCS wave function (1) is quite similar to the solution of Schrödinger equation for the parametric oscillator case, as described by a timedependent Hamiltonian [16,17].

In this case, this mechanism can be successfully implemented when, for example, a proton moves at speed $v$ in the periodic field of a crystal with period $d$. In the rest system of the proton, such motion corresponds to a nonstationary harmonic oscillator with the frequency $\Omega=v / d$. Thus, it is shown [15] that the optimum condition for CCS formation corresponds to the proton longitudinal energy $E_{\mathrm{opt}}=m v^{2} /$ $2 \approx 500 \mathrm{eV}$, and in this condition the correlation efficiency of a moving proton reaches a very large value $G=$ $1 / \sqrt{\left(1-r^{2}\right)} \approx 25000$ to the end of the third to fourth period of the crystal lattice, which leads to the generation of giant fluctuations $\delta T^{(\mathrm{min})} \geq 30 \mathrm{keV}$ of the transverse energy of the particle, thereby making the ${ }^{7} \mathrm{Li}(p, \alpha)^{4} \mathrm{He}$ reaction much more likely to occur. Nearly the same effect, but with some differences, takes place when a slow proton moves through the inhomogeneous (nonstationary in a particle rest system) field inside a single $\mathrm{Li}_{2}$ molecule.

\section{DESCRIPTION OF THE PROPOSED EXPERIMENT}

A low energy ion irradiation facility, such as an ECR Ion Source, that can accelerate $\mathrm{H}^{+}, \mathrm{D}^{+}, \mathrm{He}^{+}$, and $\mathrm{Ar}^{+}$, is required. Technical characteristics are a high current (5 mA), low energy (100-1500 eV), ultrahigh vacuum (UHV) $\left(10^{-9}-10^{-10} \mathrm{mbar}\right)$, with one heating stage. To provide accurate measurements, the contamination of the target surface by even a few monatomic layers of carbon and oxygen, where significant energy is deposited, is to be avoided absolutely, and this can be done by argon sputtering in a UHV environment $\left(<10^{-10}\right.$ mbar $)$. Careful target cleanness monitoring should be implemented.

A LiF target will be irradiated with low energy $\mathrm{H}^{+}$ions: $200 \mathrm{eV}-1.5 \mathrm{keV}$. The target will be prepared in two versions: amorphous and crystalline [cleaved along the (100) plane, with a possible variation of angles relative to this direction], to observe any significant difference due to the crystal lattice structure. Particle incidence will be both normal and off normal, to see whether channeling or nonchanneling effects influence the cross section, as well. $\mathrm{LiF}$ being an insulator, to avoid target charging by the low energy, high-intensity proton beam, an ultrathin LiF film $(\approx 1 \mathrm{~nm})$ may be deposited on a conductive, grounded backing, where penetrating protons are collected (the proton projected range at $1 \mathrm{keV}$ is $\approx 15 \mathrm{~nm}$ ). If this were not to solve the problem, we can consider also the use of a pure lithium metal target. 

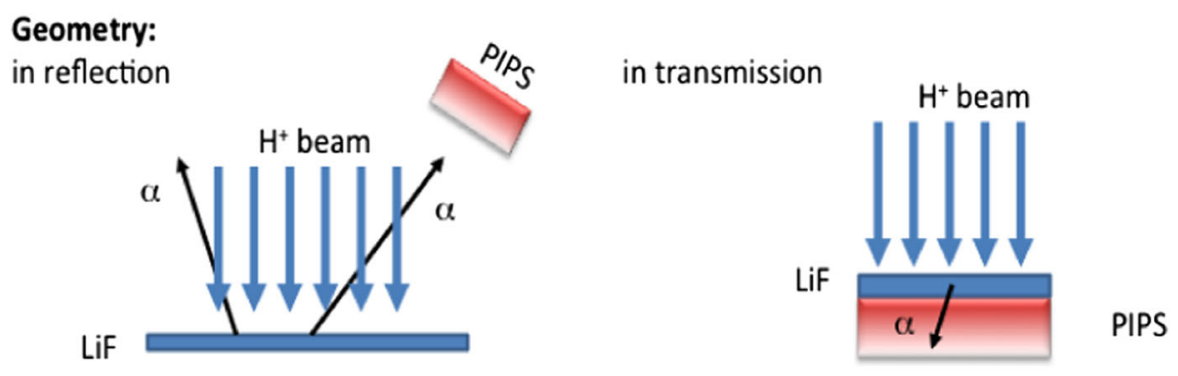

FIG. 2. Two measurement schemes (courtesy of Dr. S. Facsko, HZDR).

A sketch of the proposed setup is depicted in Fig. 2, in which also the option of having the detectors placed behind the target to intercept forward $\alpha$ particles is shown. The production of $\alpha$ particles from the nuclear reactions, ${ }^{7} \mathrm{Li}(p, \alpha){ }^{3} \mathrm{He}(Q=17.35 \mathrm{MeV})$ and ${ }^{6} \mathrm{Li}(p, \alpha)^{3} \mathrm{He}(Q=$ $4.02 \mathrm{MeV}$ ), will be measured by passivated implanted planar Si (PIPS) detectors (e.g., Canberra A series for alpha spectroscopy), $300 \mu \mathrm{m}$ thickness.

\section{EXPECTED RESULTS}

The formation of CCSs can be a general clue to all the above-mentioned effects and to other intriguing aspects of low energy nuclear reactions (LENRs), such as the absence of neutral particle (neutrons, gammas) production. Cross sections of the ${ }^{6} \mathrm{Li}(p, \alpha)^{3} \mathrm{He}$ and ${ }^{7} \mathrm{Li}(p, \alpha){ }^{4} \mathrm{He}$ reactions have been measured down to c.m. energies of $25 \mathrm{keV}$ [18] or extrapolated to $10 \mathrm{keV}$ [19], providing a measurable rate even at such low energies (see Table I below, where D-D fusion cross section estimates at $1 \mathrm{keV}$ are shown for comparison). The astrophysical factor $S(0)$ represents the strong nuclear force term at zero energy, after subtraction of the Coulomb repulsive term, and is used for extrapolation purposes.

In the last (sixth) column, a value of $r=0.99995$, equivalent to $G=1 / \sqrt{\left(1-r^{2}\right)}=100$, for the correlation coefficient is assumed, and the cross section at $1 \mathrm{keV}$ resulting from the transmissivity enhancement of high momentum spread particles as computed by Eq. (2) is compared with the uncorrelated usual case $(r=0)$ in column 5 .

The cross section for the two reactions of interest exhibits a strong dependence on the momentum variance $\sigma_{p}$, not on the mean value of momentum ( $a$ is a constant), and should increase by more than 32 orders of magnitude for maximum correlation.

Clearly, the reaction rate will depend on the correlation factor $r$, which cannot be predicted at this level, also because no previous measurement was ever done at such low energies $(\leq 1 \mathrm{keV})$.

Very preliminary calculations [15] give a correlation factor of $r \geq 0.999$, corresponding to $\sigma \approx 1 \mathrm{mb}$, to get a measurable counting rate, even with a small angular acceptance detector.

Because of the specificity of LENRs originated from giant energy fluctuations, the CCS model shows that, of the two possible reactions $\left[{ }^{7} \mathrm{Li}(p, \alpha)^{4} \mathrm{He}\right.$ and ${ }^{6} \mathrm{Li}(p, \alpha)^{3} \mathrm{He}$, only the reaction with participation of the ${ }^{7} \mathrm{Li}$ isotope should be realized. The reason for this phenomenon is the following. The duration of this reaction is quite short: $t_{\text {reac }} \approx 10^{-20} \mathrm{sec}$ as compared to the existence time of the energy fluctuations in a coherent correlated state of $\delta t \approx 10^{-17} \ldots 10^{-18} \mathrm{sec}$. On the other hand, the duration $t_{\text {reac }} \approx 10^{-13} \mathrm{sec}$ of the reaction with the participation of the lighter ${ }^{6} \mathrm{Li}$ isotope is substantially larger than the value $\delta t$, which makes this synthesis channel impossible [12]. One of the goals of such experiments will be testing these important aspects, which would be consistent with our theoretical model.

\section{CONCLUSIONS}

This work describes a simple and quite feasible experiment to definitely verify the existence of coherent correlated states, which should be a powerful interpretation tool to explain a number of paradoxes in low energy nuclear physics without introducing complicated and groundless heuristic models. It must be stressed that this is the very first use of a particle beam to investigate such topics.

TABLE I. Actual values and expected increase of significant parameters for some relevant reactions.

\begin{tabular}{lccccc}
\hline \hline Reaction & $\sigma\left(\mathrm{E}_{\mathrm{cm}}=10 \mathrm{keV}\right) \mathrm{b}$ & $\mathrm{S}(0) \mathrm{keV} \times \mathrm{b}$ & $\mathrm{E}_{G}{ }^{1 / 2}(\mathrm{keV})^{1 / 2}$ & $\sigma\left(\mathrm{E}_{\mathrm{cm}}=1 \mathrm{keV}\right) \mathrm{b}$ & $\sigma\left(\mathrm{E}_{\mathrm{cm}}=1 \mathrm{keV}\right) G=10^{2}$ \\
\hline${ }^{6} \mathrm{Li}(\mathrm{p}, \alpha)^{3} \mathrm{He}$ & $6 \times 10^{-10}$ & $5.5 \times 10^{3}$ & 87.2 & $7.4 \times 10^{-35}$ & 0.026 \\
${ }^{7} \mathrm{Li}(p, \alpha)^{4} \mathrm{He}$ & $4.3 \times 10^{-12}$ & 80 & 88.1 & $4.3 \times 10^{-37}$ & 0.029 \\
${ }^{2} \mathrm{H}(\mathrm{d}, \mathrm{p})^{3} \mathrm{H}$ & $2.81 \times 10^{-4}$ & 56 & 31.4 & $1.3 \times 10^{-12}$ & 0.281 \\
${ }^{2} \mathrm{H}(\mathrm{d}, \mathrm{n})^{3} \mathrm{He}$ & $2.78 \times 10^{-4}$ & 54 & 31.4 & $1.4 \times 10^{-12}$ & 0.281 \\
\hline \hline
\end{tabular}


Several nuclear processes, which have been deemed independent so far and as such reported on in the quoted literature, should find a unified description.

The main issue in this experiment should be the atomic cleanness of the target surface, since the useful interactions are predicted to occur in the very first monatomic layers of the crystal lattice, but there is already broad experience on this topic by other experiments.

From the present experimental state of the art, as described above, the observation of a clear $\alpha$ signal above the background with good statistical significance is expected. If so, an intensive campaign of measurements (excitation function vs energy, counting rate dependence on the impact angle, crystal vs amorphous target effects, background sources, etc.) would be then required to a first characterization of the observed phenomena.

\section{ACKNOWLEDGMENTS}

Very useful discussions with Dr. S. Facsko (HelmholtzZentrum Dresden-Rossendorf, HZDR) and Prof. K. Czerski (University of Szczecin) are gratefully acknowledged.

[1] F. Raiola et al., Enhanced electron screening in d(d, p)t for deuterated Ta*, Eur. Phys. J. A 13, 377 (2002); G. Fiorentini, C. Rolfs, F. L. Villante, and B. Ricci, Fusion rate enhancement due to energy spread of colliding nuclei, Phys. Rev. C 67, 014603 (2003); J. Kasagi, H. Yuki, T. Baba, T. Noda, J. Taguchi, M. Shimokawa, and W. Galster, Strongly enhanced $\mathrm{Li}+\mathrm{D}$ reaction in $\mathrm{Pd}$ observed in deuteron bombardment on PdLix with energies between 30 and 75 keV, J. Phys. Soc. Jpn. 73, 608 (2004).

[2] K. Czerski, D. Weissbach, A. I. Kilic, G. Ruprecht, A. Huke, M. Kaczmarski, N. Targosz-Ślęczka, and K. Maass, Screening and resonance enhancements of the $2 \mathrm{H}(\mathrm{d}, \mathrm{p}) 3 \mathrm{H}$ reaction yield in metallic environments, Eur. Phys. Lett. 113, 22001 (2016), and references therein.

[3] K. Czerski (private communication).

[4] S. Lipinski and H. Lipinski, Hydrogen-lithium fusion device, Patent No. WO 2014/189799 A9, 2013.

[5] V. I. Vysotskii, M. V. Vysotskyy, and S. Bartalucci, Features of the formation of correlated coherent states and nuclear fusion induced by the interaction of slow particles with crystals and free molecules, J. Exp. Theor. Phys. 127, 479 (2018), and references therein.

[6] A. V. Dodonov and V. V. Dodonov, Tunneling of slow quantum packets through the high Coulomb barrier, Phys. Lett. A 378, 1071 (2014).

[7] V. V. Dodonov, E. V. Kurmyshev, and V. I. Man'ko, Generalized uncertainty relation and correlated coherent states, Phys. Lett. 79A, 150 (1980).

[8] W. Heisenberg, Über den anschaulichen inhalt der quantentheoretischen kinematik und mechanik, Z. Phys. 43, 172 (1927).

[9] E. H. Kennard, Zur quantenmechanik einfacher bewegungstypen, Z. Phys. 44, 326 (1927).

[10] H. P. Robertson, The Uncertainty Principle, Phys. Rev. 34, 163 (1929).

[11] E. Schrödinger, Zum Heisenbergschen unschärfeprinzip, Ber. Kgl. Akad. Wiss., Berlin, S 24, 296 (1930); an English translation can be found at: arXiv:quant-ph/9903100.

[12] V. I. Vysotskii and M. V. Vysotskyy, Universal mechanism of realization of nuclear reactions at low energy, RENSIT 9, 21 (2017).

[13] V. I. Vysotskii and M. V. Vysotskyy, Formation of correlated states and tunneling for a low energy and controlled pulsed action on particles, J. Exp. Theor. Phys. 125, 195 (2017).

[14] R. J. Glauber, The Quantum Theory of Optical Coherence, Phys. Rev. 130, 2529 (1963).

[15] D. F. Walls, Squeezed states of light, Nature (London) 306, 141 (1983).

[16] V. V. Dodonov, A. B. Klimov, and V. I. Man'ko, Physical significance of Correlated And Squeezed States, Group Theoretical Methods in Physics, Proceedings of the XVIII International Colloquium Held at Moscow, USSR, 1990, Lecture Notes in Physics Vol. 382 (Springer, Heidelberg, 1990), p. 450.

[17] V. I. Vysotskii and M. V. Vysotskyy, Coherent correlated states and low-energy nuclear reactions in non stationary systems, Eur. Phys. J. A 49, 99 (2013), and references therein.

[18] C. Rolfs and R. W. Kavanagh, The ${ }^{7} \mathrm{Li}(p, \alpha)^{4} \mathrm{He}$ cross section at low energies, Nucl. Phys. A455, 179 (1986).

[19] G. Calvi et al., Indirect measurement of nuclear reaction cross section at astrophysical energies, Nucl. Phys. A621, 139 (1997). 